BULL. AUSTRAL. MATH. SOC.

VOL. 30 (1984), 1-9.

\title{
FIXED POINT THEOREMS BY ALTERING DISTANCES BETWEEN THE POINTS
}

\author{
M.S. Khan, M. SWaleh and S. Sessa
}

In this paper we have established some fixed point theorems in complete and compact metric spaces.

\section{Introduction}

Let $R^{+}$be the set of nonnegative real numbers and $N$ the set of positive integers.

Delbosco [1] and skof [8] have established fixed point theorems for selfmaps of complete metric spaces by altering the distances between the points with the use of a function $\varphi: R^{+} \rightarrow R^{+}$satisfying the following properties:

1. $\varphi$ is continuous and strictly increasing in $R^{+}$;

2. $\varphi(t)=0$ if and only if $t=0$;

3. $\varphi(t) \geqslant M \cdot t^{\mu}$ for every $t>0$, where $M>0, \mu>0$ are constant. We denote the set of above functions $\varphi$ with $\Phi$.

Precisely in [8, corol. 2] the following theorem was proved:

THEOREM 1. Let $T$ be a seifmap of a complete metric space $(X, d)$ and $\varphi \in \Phi$ such that for every $x, y$ in $X$, (A)

$$
\varphi(d(T x, T y)) \leqslant a \cdot \varphi(d(x, y))+b \cdot \varphi(d(x, T x))+c \cdot \varphi(d(y, T y))
$$

Received 5 January 1984.

Copyright Clearance Centre, Inc. Serial-fee code: 0004-9727/84 $\$ A 2.00+0.00$ 
where $0 \leqslant a+b+c<1$. Then $T$ has a unique fixed point.

In [1], the author has considered functions $\varphi \in \Phi$ such that $\varphi(t)=t^{n}, n \in N$, for every $t \geqslant 0$. $\varphi(t)=t^{2}$

REMARK 1. Note that $\varphi$ is not necessarily a metric: for example,

REMARK 2. By symmetry of metric $d$, we may assume $b=c$ in $(A)$. The purpose of this paper is to study a stronger condition than $(A)$ and to remove the hypothesis (3) which seems superfluous. Furthermore, our main theorem is an improvement upon some fixed point theorems of Rakotch [5], Reich [6], and a result of Fisher [3] in compact metric spaces. other related results can be found in sessa [7].

\section{Main theorem}

We shall prove a fixed point theorem offering a condition closely related to that used by Massa [4] in Banach spaces. Strictly speaking, the following theorem holds:

THEOREM 2. Let $(X, d)$ be a complete metric space, $T$ a selfmap of $X$, and $\varphi: R^{+} \rightarrow R^{+}$an increasing, continuous function satisfying property (2). Furthermore, let $a, b, c$ be three decreasing functions from $R^{+} \backslash\{0\}$ into $[0,1\}$ such that $a(t)+2 b(t)+c(t)<1$ for every $t>0$. Suppose that $T$ satisfies the following condition:

$$
\varphi(d(T x, T y)) \leqslant a(d(x, y)) \cdot \varphi(d(x, y))+b(d(x, y)) \cdot\{\varphi(d(x, T x))+
$$

$$
\varphi(d(y, T y)\}+c(d(x, y)) \cdot \min \{\varphi(d(x, T y)), \varphi(d(y, T x))\},
$$

where $x, y \in X$ and $x \neq y$. Then $T$ has a unique fixed point.

Proof. Let $x_{0}$ be a point of $X$. We define

(*) $\quad x_{n+1}=T x_{n}, \quad \tau_{n}=d\left(x_{n}, x_{n+1}\right)$, for all $n \in N \cup\{0\}$.

We first prove that $T$ has a fixed point. We may assume $\tau_{n}>0$ for each n. From (B), we obtain:

$$
\begin{gathered}
\varphi\left(\tau_{n+1}\right) \leqslant a\left(\tau_{n}\right) \cdot \varphi\left(\tau_{n}\right)+b\left(\tau_{n}\right) \cdot\left\{\varphi\left(\tau_{n}\right)+\varphi\left(\tau_{n+1}\right)\right\}+ \\
c\left(\tau_{n}\right) \cdot \min \left\{\varphi\left(d\left(x_{n}, x_{n+2}\right)\right), \varphi\left(d\left(x_{n+1}, x_{n+1}\right)\right)\right\} .
\end{gathered}
$$


Hence we obtain:

$$
\varphi\left(\tau_{n+1}\right) \leqslant \frac{a\left(\tau_{n}\right)+b\left(\tau_{n}\right)}{1-b\left(\tau_{n}\right)} \cdot \varphi\left(\tau_{n}\right)<\varphi\left(\tau_{n}\right) .
$$

Since $\varphi$ is increasing, $\left\{\tau_{n}\right\}$ is a decreasing sequence.

We put $\lim _{n \rightarrow \infty} \tau_{n}=\tau$ and suppose that $\tau>0$. By $(2.1)$, then $\tau_{n} \geqslant \tau$ implies that

$$
\varphi\left(\tau_{n+1}\right) \leqslant \frac{a(\tau)+b(\tau)}{1-b(\tau)} \cdot \varphi\left(\tau_{n}\right)
$$

By letting $n \rightarrow \infty$, since $\varphi$ is continuous, we have:

$$
\varphi(\tau) \leqslant \frac{a(\tau)+b(\tau)}{1-b(\tau)} \cdot \varphi(\tau)<\varphi(\tau)
$$

which is inadmissible. So $\tau=0$. Now we prove that $\left\{x_{n}\right\}$ is a cauchy sequence. Suppose it is not. Then there exist $\varepsilon>0$ and two sequences $\{p(n)\},\{q(n)\}$ such that for every $n \in N \cup\{0\}$, we find that $p(n)>q(n) \geqslant n, d\left(x_{p(n)}, x_{q(n)}\right) \geqslant \varepsilon$ and $d\left(x_{p(n)-1}, x_{q(n)}\right)<\varepsilon$.

For each $n \geqslant 0$, we put $s_{n}=d\left(x_{p(n)}, x_{q(n)}\right)$. Then we have $\varepsilon \leqslant s_{n} \leqslant d\left(x_{p(n)-1}, x_{p(n)}\right)+d\left(x_{p(n)-1}, x_{q(n)}\right)<\tau_{p(n)-1}+\varepsilon$.

Since $\left\{\tau_{n}\right\}$ converges to $0,\left\{s_{n}\right\}$ converges to $\varepsilon$.

Furthermore, the triangular inequality implies, for each $n \geqslant 0$, $-\tau_{p(n)}-\tau_{q(n)}+s_{n} \leqslant d\left(x_{p(n)+1}, x_{q(n)+1}\right) \leqslant \tau_{p(n)}+\tau_{q(n)}+s_{n^{\prime}}$ and therefore also the sequence $\left\{d\left(x_{p(n)+1}, x_{q(n)+1}\right)\right\}$ converges to $\varepsilon$. From (B), we also deduce:

$$
\begin{aligned}
& \varphi\left(d\left(x_{p(n)+1}, x_{q(n)+1}\right)\right) \leqslant a\left(s_{n}\right) \cdot \varphi\left(s_{n}\right)+b\left(s_{n}\right) \cdot\left\{\varphi\left(\tau_{p(n)}\right)+\varphi\left(\tau_{q(n)}\right)\right\}+ \\
& c\left(s_{n}\right) \cdot \min \left\{\varphi\left(d\left(x_{p(n)}, x_{q(n)+1}\right)\right), \varphi\left(d\left(x_{q(n)}, x_{p(n)+1}\right)\right)\right\} \leqslant a(\varepsilon) \cdot \varphi\left(s_{n}\right)+ \\
& b(\varepsilon) \cdot\left\{\varphi\left(\tau_{p(n)}\right)+\varphi\left(\tau_{q(n)}\right)\right\}+c(\varepsilon) \cdot \varphi\left(s_{n}+\tau_{q(n)}+\tau_{p(n)}\right) .
\end{aligned}
$$


For $n \rightarrow \infty$ we are left with

$$
\varphi(\varepsilon) \leqslant\{a(\varepsilon)+c(\varepsilon)\} \cdot \varphi(\varepsilon)<\varphi(\varepsilon),
$$

which is absurd. Therefore $\left\{x_{n}\right\}$ is a Cauchy sequence. By completeness of $X,\left\{x_{n}\right\}$ converges to some point $z$. Now we show that $z$ is a fixed point of $T$. Since each $\tau_{n}>0$, there is a subsequence $\left\{x_{h(n)}\right\}$ of $\left\{x_{n}\right\}$ such that $x_{h(n)} \neq z$ for each $n \geqslant 0$ and we put $\rho_{n}=d\left(z, x_{n}\right)$. Since $b<1 / 2$, we obtain from (B):

$$
\begin{aligned}
& \varphi\left(d\left(x_{h(n)+1}, T z\right)\right) \leqslant a\left(\rho_{h(n)}\right) \cdot \varphi\left(\rho_{h(n)}\right)+b\left(\rho_{h(n)}\right) \cdot\left\{\varphi\left(\tau_{h(n)}\right)+\varphi(d(z, T z))\right\} \\
& +c\left(\rho_{h(n)}\right) \cdot \min \left\{\varphi\left(\rho_{h(n)+1}\right), \varphi\left(d\left(x_{h(n)}, T z\right)\right)\right\} \\
& <\varphi\left(\rho_{h(n)}\right)+1 / 2\left\{\varphi\left(\tau_{h(n)}\right)+\varphi(d(z, T z))\right\}+\varphi\left(\rho_{h(n)}+\tau_{h(n)}\right) .
\end{aligned}
$$

Since $\left\{\rho_{n}\right\}$ converges to 0 , for $n \rightarrow \infty$ the last inequality yields

$$
\underset{n \rightarrow \infty}{\limsup } \varphi\left(d\left(x_{h(n)+1}, T z\right)\right) \leqslant 1 / 2 \varphi(d(z, T z)) .
$$

on the other hand, the triangular inequality implies that

$$
d(z, T z) \leqslant \rho_{h(n)}+\tau_{h(n)}+d\left(x_{h(n)+1}, T z\right),
$$

which in turn implies that

$$
\varphi(d(z, T z)) \leqslant \underset{n \rightarrow \infty}{\limsup } \varphi\left(d\left(x_{h(n)+1}, T z\right)\right)
$$

From (2.2) and (2.3), then we deduce

$$
\varphi(d(z, T z)) \leqslant 1 / 2 \varphi(d(z, T z))
$$

that is, $\varphi(d(z, T z))=0$ and therefore $d(z, T z)=0$.

If $T$ has two distinct fixed points $x, y$ in $X$, then $\varphi(d(x, y))=\varphi(d(T x, T y)) \leqslant\{a(d(x, y))+c(d(x, y))\} \cdot \varphi(d(x, y))<\varphi(d(x, y))$, a contradiction. This completes the proof.

REMARK 3. Note that we have not supposed the continuity of $T$. 
3. Some consequences and examples

If we assume $c=0$ in Theorem 2 and take $a, b$ as constants, we obtain Theorem 1. The following examples show that condition (B) is more general than condition (A) :

EXAMPLE 1. Let $X$ be the subset of $R^{2}$ defined by

$$
X=\{A, B, C, D, E\},
$$

where $A \equiv(-1,0), B \equiv(0,0), C \equiv(0,1 / 2), D \equiv(0,1), E \equiv(-1,1)$.

Let $T: X \rightarrow X$ be given by

$$
T(A)=B, T(B)=T(C)=T(D)=C, T(E)=D
$$

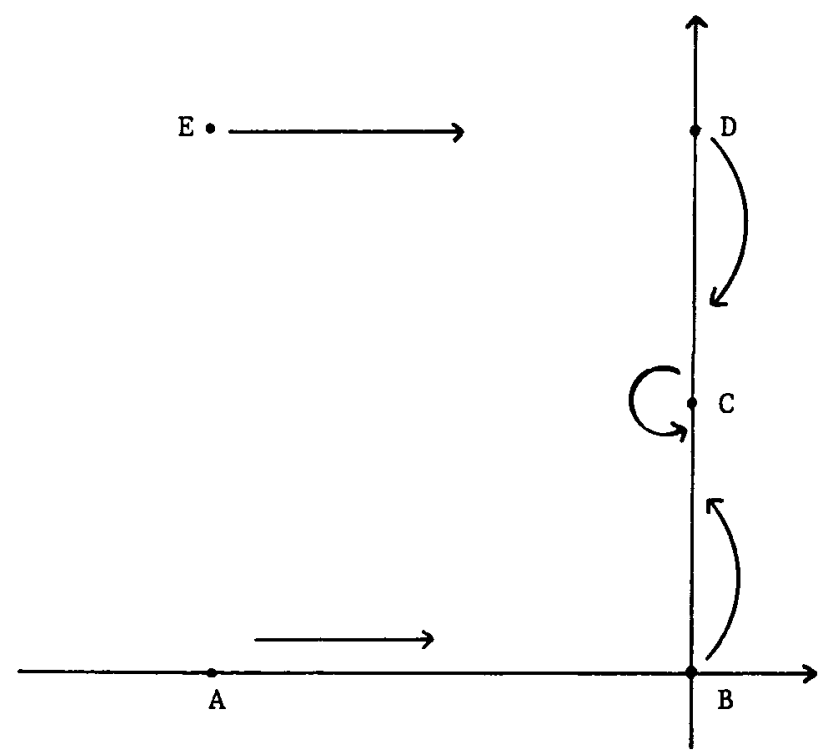

Then $T$ satisfies condition (B) by letting:

$$
a(t)=3 / 4, b(t)=0, c(t)=1 / 5 \text { and } \varphi(t)=t^{2} \text { for any } t \in R^{+} \text {. }
$$

However, $T$ does not satisfy condition (A). For otherwise, choosing $x=A$ and $y=E$, we would have

$$
\varphi(d(T A, T E))=\varphi(1) \leqslant a \cdot \varphi(1)+b \cdot \varphi(1)+c \cdot \varphi(1)<\varphi(1),
$$

which is a contradiction. 
In Theorem 2 if we assume $c=0$ and $\varphi(t)=t$ for every $t \geqslant 0$, we obtain the following condition indebted to Reich [6],

(C) $\quad d(T x, T y) \leqslant a(d(x, y)) \cdot d(x, y)+b(d(x, y)) \cdot\{d(x, T x)+d(y, T y)\}$.

The example given below proves that condition (B) is more general than condition (C) :

EXAMPLE 2. Consider the set $X=\{1,2,3,4\}$ equipped with the metric $d$ which is defined by

$$
\begin{array}{lll}
d(1,2)=2 / 5, & d(1,3)=1 / 5, & d(1,4)=3 / 5, \\
d(2,3)=2 / 5, & d(2,4)=1, & d(3,4)=\sqrt{2} / 2 .
\end{array}
$$

Let $T$ be a selfmap of $X$ such that

$$
T(1)=T(3)=T(4)=3, \quad T(2)=4 .
$$

Here all the assumptions of Theorem 2 are satisfied with

$$
a(t)=1 / 16, b(t)=1 / 3, c(t)=1 / 16 \text { and } \varphi(t)=t^{4} \text { for any } t \in R^{+} \text {. }
$$

But the condition (C) is not fulfilled, otherwise for $x=1$ and $y=2$, and all functions $a, b$ from $R^{大} \backslash\{0\}$ into $[0,1 l$ with $a+2 b<1$, we would have

$d(T 1, T 2)=\sqrt{2} / 2 \leqslant a(2 / 5) .2 / 5+b(2 / 5) .6 / 5 \leqslant a(2 / 5) .3 / 5+2 b(2 / 5) .3 / 5<3 / 5$, which is a contradiction as $\sqrt{2} / 2>3 / 5$.

If we assume $b=c=0$ in Theorem 2, we get the following:

THEOREM 3. Let $(X, d)$ be a complete metric space, $T$ a selfmap of $X$ and $\varphi: R^{+} \rightarrow R^{+}$be an increasing, continuous function for which property (2) holds. Let $a$ be a decreasing function from $R^{+} \backslash\{0\}$ into 10,11 such that

$$
\varphi(d(T x, T y)) \leqslant a(d(x, y)) . \varphi(d(x, y)),
$$

where $x, y \in X$ and $x \neq y$. Then $T$ has a unique fixed point.

REMARK 4. For $\varphi(t)=t$ Theorem 3 yields Rakotch's fixed point theorem [5]. 


\section{A result in compact metric spaces}

In a paper of Fisher [3], the following theorem has been given: THEOREM 4. Let $T$ be a continuous selfmap of a compact metric space $(X, d)$ such that

$$
d(T x, T y)<1 / 2\{d(x, T x)+d(y, T y)\}
$$

for all distinct $x, y$ in $X$. Then $T$ has a unique fixed point.

Following the fundamental idea of our work presented in section 2 we now generalize Theorem 4 as follows:

THEOREM 5. Let $T$ be a continuous selfmap of a metric space $(X, d)$ such that for some $x_{0} \in X$ the sequence $\left\{T^{n} x_{0}\right\}$ has a cluster point $z \in X$. Let there exist a continuous function $\varphi: R^{+} \rightarrow R^{+}$satisfying property (2). Furthermore, for all distinct $x, y$ in $X$ the inequality

$$
\varphi(d(T x, T y))<c . \varphi(d(x, y))+\left(\frac{1-c}{2}\right)\{\varphi(d(x, T x))+\varphi(d(y, T y))\}
$$

holds, where $0 \leqslant c \leqslant 1$. Then $z$ is the unique fixed point of $T$.

Proof. If $T^{n} x_{0}=T^{n+1} x_{0}$ for some $n \in N$, then $z=T^{k} x_{0}$ for all $k \geqslant n$ and therefore the thesis. So we may assume that $T^{n} x_{0} \neq T^{n+1} x_{0}$ for every $n \in N$. Let $\{k(n)\}$ be a sequence of positive integers such that $\left\{p^{k(n)} x_{0}\right\}$ converges to $z$. By maintaining the notations $(*)$ of Theorem 2, and using the continuity of $T$, we have

$$
\lim _{n \rightarrow+\infty} x_{k(n)+1}=T(z) \text { and } \lim _{n \rightarrow+\infty} x_{k(n)+2}=T^{2}(z)
$$

As $\varphi$ is continuous, it also follows that

$$
\varphi(d(z, T z))=\lim _{n \rightarrow+\infty} \varphi\left(\tau_{k(n)}\right)=\lim _{n \rightarrow+\infty} \varphi\left(\tau_{k(n)+1}\right)=\varphi\left(d\left(T z, T^{2} z\right)\right)
$$

Now we claim that $z=T z$, otherwise, by condition (F) when $x=z$ and $y=T z$, we have

$$
\varphi\left(d\left(T z, T^{2} z\right)\right)<c . \varphi(d(z, T z))+\frac{1-c}{2}\left\{\varphi(d(z, T z))+\varphi\left(d\left(T z, T^{2} z\right)\right)\right\}
$$


This last inequality implies that

$$
\varphi\left(d\left(T z, T^{2} z\right)\right)<\varphi(d(z, T z)),
$$

which contradicts (4.1).

Property (2) assures the uniqueness of the fixed point.

REMARK 5. If $\varphi(t)=t$ for any $t \geqslant 0$ and $c=1$, Theorem 5 becomes a well-known result of Edelstein [2].

REMARK 6. If $\varphi(t)=t$ for any $t \geqslant 0$ and $c=0$, Theorem 5 reduces to Theorem 4 as every sequence in a compact metric space necessarily has a cluster point.

Using the following example, we show that condition (F) is more general than condition $(E)$ :

EXAMPLE 3. Consider the set $X=\{1,2,3,4\}$ with the metric $d$ defined as

$$
\begin{array}{lll}
d(1,2)=9 \sqrt{3}, & d(1,3)=3 \sqrt{3}, & d(1,4)=12 \sqrt{3}, \\
d(2,3)=9 \sqrt{3}, & d(2,4)=21 \sqrt{3}, & d(3,4)=21 .
\end{array}
$$

Let $T: X \rightarrow X$ be defined by

$$
T(1)=T(3)=T(4)=3, T(2)=4 \text {. }
$$

Then condition $(F)$ is clearly verified for $\varphi(t)=t^{2}$ and $c=1 / 3$. But condition (E) does not hold because for $x=1$ and $y=2$, we have:

$$
d(T 1, T 2)=21>12 \sqrt{3}=\frac{1}{2}(3 \sqrt{3}+21 \sqrt{3})=\frac{1}{2}\{d(1, T 1)+d(2, T 2)\} .
$$

The idea of this example appears in [1].

Thanks are due to Professors B. Fisher and C. Zanco, who in private communications, have supplied some kind suggestions about Theorem 5 and Example 1 of this note.

\section{References}

[1] D. Delbosco, "Un'estensione di un teorema sul punto fisso di s. Reich", Rend. Sem. Mat. Univers. Politeon. Torino, 35 (1976-77), 233-238. 
[2] M. Edelstein, "On fixed and periodic points under contractive mappings", J. London Math. Soc., 37 (1962), 74-79.

[3] B. Fisher, "A fixed point mapping", Bull. Calcutta Math. Soc. 68 $(19.76), 265-266$.

[4] S. Massa, "Un'osservazione su un teorema di Browder-Roux-Soardi", BoZ2. U.M.I., (4) 7 (1973), 151-155.

[5] E. Rakotch, "A note on contractive mappings", Proc. Amer. Math. Soc., 13 (1962), 459-465.

[6] S. Reich, "Kannan's fixed point theorem", Boll. U.M.I. (4) 4 (1971), 1-11.

[7] S. Sessa, "Un teorema orbitale di punto fisso ed una sua conseguenza", Le Matematiche, Catania (Italy), to appear.

[8] F. Skof, "Teorema di punti fisso per applicazioni negli spazi metrici", Atti. Accad. Sci. Torino, 111 (1977), 323-329.

M. S. Khan,

Department of Mathematics,

Aligarh Muslim University,

A1igarh - 202001,

India ;

M. Swaleh,

Department of Mathematics,

Aligarh Muslim University,

Aligarh - 202001,

India;
S. Sessa,

Institute of Mathematics,

Faculty of Architecture, University of Naples,

Via Monteoliveto 3,

Naples 80134,

Italy. 